\title{
AN OPTIMUM IMPLICIT RECURRENCE FORMULA FOR THE HEAT CONDUCTION EQUATION*
}

\section{BY STEPHEN H. CRANDALL (Massachusetts Institute of Technology)}

The equation $\psi_{x x}=\psi_{t}$ may be approximately integrated over a finite difference network, $\Delta x=h, \Delta t=r h^{2}$, using

$$
\begin{aligned}
\psi_{i, k+1}-r \theta\left(\psi_{i-1, k+1}-2 \psi_{i, k+1}+\right. & \left.\psi_{i+1, k+1}\right) \\
& =\psi_{i, k}+r(1-\theta)\left(\psi_{i-1, k}-2 \psi_{i, k}+\psi_{i+1, k}\right),
\end{aligned}
$$

where $\psi_{i, k}=\psi(j \Delta x, k \Delta t)$ and $0 \leq \theta \leq 1$. When $\theta=0$, we have the recurrence formula of Schmidt [1], when $\theta=1 / 2$ that of Crank and Nicolson [2] and when $\theta=1$ a formula mentioned by O'Brien, Hyman and Kaplan [3]. The truncation error of (1) is ordinarily $0\left(h^{2}\right)$. Milne [4] has shown that when $\theta=0$ the truncation error is $0\left(h^{4}\right)$ for $r=1 / 6$. Fowler showed that the stability limit for $\theta=0$ was in the neighborhood of $r=1 / 2$; the exact value depending on the number of spatial subdivisions and the boundary conditions. It is also known [6] that for $\theta=0$ some of the finite difference modes have oscillatory time behavior when $r$ is greater than about $1 / 4$.

It is a straightforward matter to extend these analyses to $0 \leq \theta \leq 1$. The results are shown in Fig. 1. Each point in the $(r, \theta)$ plane represents a recurrence formula (1). All are implicit except those with $\theta=0$. The loci of the stability and oscillation limits

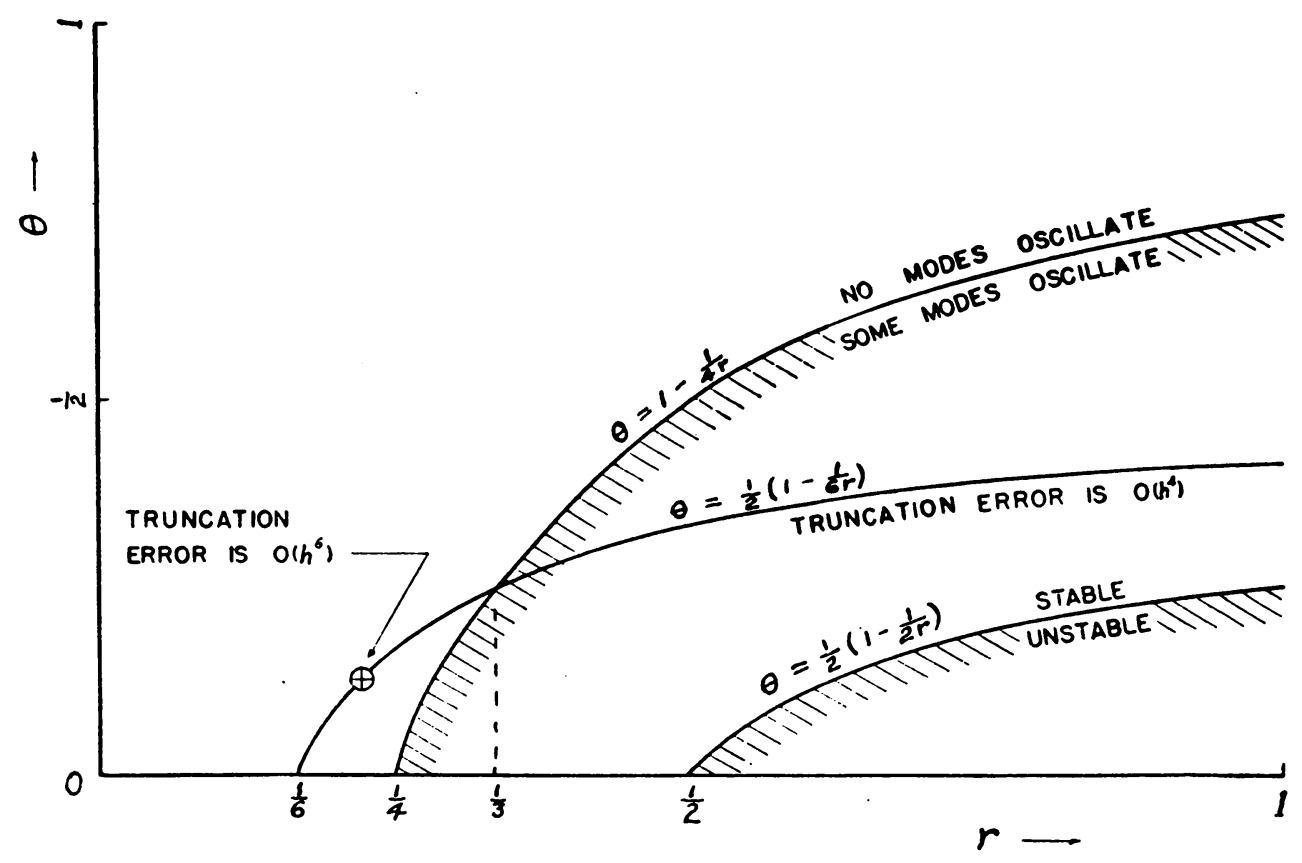

FIG. 1

${ }^{*}$ Received November 10, 1954. 
are shown along with the family of formulas for which the truncation error is $0\left(h^{4}\right)$. The derivation of these loci is outlined below.

The general solution of (1) in a domain where the boundary conditions at $j=0$ and $j=M$ are independent of $k$ can be obtained by the method of separation of variables [7] as a sum of products of space modes multiplied by time factors. The space modes are determined by the eigenvalue equation

$$
X_{i+1}-(2-\lambda) X_{i}+X_{i-1}=0
$$

and the boundary conditions. The number of modes depends on the number of spatia ${ }^{l}$ subdivisions $M$. The most oscillatory mode has the largest eigenvalue $\lambda_{\max }$. It is not difficult to show that $\lambda_{\max } \rightarrow 4$ as $M \rightarrow \infty$. Its precise value for finite $M$ depends on $M$ and also on what finite difference approximations [8] have been used at the boundaries.

The time factors $T_{k}$ which multiply the space modes $X_{j}$ are

$$
T_{k}=\left(\frac{1-(1-\theta) \lambda r}{1+\theta \lambda r}\right)^{k}
$$

For (1) to be stable in the sense that no solution can become unbounded with increasing $k$ it is necessary for the absolute value of the fraction in (3) to be less than unity for all modes. To obtain the stability limit we set this fraction equal to -1 and insert $\lambda_{\max }$. Using $\lambda_{\max }=4$ we obtain the locus

$$
\theta=\frac{1}{2}\left(1-\frac{1}{2 r}\right)
$$

shown in Fig. 1 which is the limiting stability locus as $M \rightarrow \infty$.

For all modes to have non-oscillatory time behaviour it is necessary for the fraction in (3) to be non-negative. To obtain the oscillation limit we set the numerator of this fraction equal to zero and insert $\lambda_{\max }$. Again using $\lambda_{\max }=4$ we obtain the locus

$$
\theta=1-\frac{1}{4 r}
$$

shown in Fig. 1.

To investigate the truncation error of (1) the values of an exact solution to $\psi_{x x}=\psi_{\mathrm{t}}$ at neighboring net points are represented by means of Taylor's series centered at $(j \Delta x, k \Delta t)$. When these are substituted in (1) the remainder terms of the Taylor's series are

$$
h^{2}\left(\frac{r}{2}-\frac{1}{12}-r \theta\right) \psi_{\iota 1}+h^{4}\left(\frac{r^{2}}{6}-\frac{1}{360}-\frac{r^{2} \theta}{2}-\frac{r \theta}{12}\right) \psi_{\iota 1,}+0\left(h^{6}\right),
$$

where we have used the identities

$$
\psi_{t t}=\psi_{t x x}=\psi_{x x x x} \quad \psi_{t t t}=\psi_{t 1 x x}=\psi_{t x x x x}=\psi_{x x x x x x} .
$$

Thus the truncation error is ordinarily $0\left(h^{2}\right)$ but is $0\left(h^{4}\right)$ when

$$
\theta=\frac{1}{2}\left(1-\frac{1}{6 r}\right) \text {. }
$$


Among the sets of values which satisfy (8) there is one set which also makes the coefficient of $h^{4}$ in (6) vanish. It is easily verified that this set is

$$
\begin{aligned}
& r=\frac{(5)^{1 / 2}}{10}=0.2236, \\
& \theta=\frac{3-(5)^{1 / 2}}{6}=0.1273 .
\end{aligned}
$$

The truncation error of (1) with the particular values (9) is therefore $0\left(h^{6}\right)$. This should provide a superior recurrence formula for integrating $\psi_{x x}=\psi_{t}$. As usual with implicit formulas it will be necessary to invert but this can be done once and for all as soon as the number of spatial subdivisions has been chosen.

\title{
REFERENCES
}

1. E. Schmidt, Über die Andwendung der Differenzenrechnung auf technische Anheiz-und-Abkühungsprobleme, A. Föppl Festschrift, Springer, Berlin, 1924, pp. 179-189

2. J. Crank and P. Nicolson, A practical method for numerical evaluation of solutions of partial differential equations of heat-conduction type, Proc. Camb. Phil. Soc. 32, 50-67 (1947)

3. G. G. O'Brien, M. A. Hyman and S. Kaplan, A study of the numerical solution of partial differential equations, J. Math. Phys. 29, 223-251 (1951)

4. W. E. Milne, Numerical solution of differential equations, John Wiley, N. Y., 1953, p. 122

5. C. M. Fowler, Analysis of numerical solutions of transient heat-flow problems, Quart. Appl. Math. 3, 361-376 (1946)

6. S. H. Crandall, Implicit vs. explicit recurrence formulas for the linear diffusion equation. J. Assoc. Comput. Mach. 2, 42-49 (1955)

7. See, for example, F. B. Hildebrand, Methods of applied mathematics, Prentice-Hall, N. Y., 1952, p. 330

8. For six possible alternatives see P. H. Price and M. R. Slack, Stability and accuracy of numerical solutions of the heat flow equation, Brit. J. Appl. Phys. 3, 379-384 (1952)

\section{ON A METHOD OF GENERATING THREE-DIMENSIONAL POTENTIAL FLOWS FROM TWO-DIMENSIONAL ONES*}

\author{
By CHIA-SHUN YIH (Ioma Institute of Hydraulic Research)
}

In 1951, A. Wintner ${ }^{1}$ presented a method of deriving three-dimensional potential flows from two-dimensional ones. This method was found by Weingarten ${ }^{2}$ over sixty years ago and rediscovered by Levi-Civita ${ }^{3}$ a decade later. Whereas it is surprising that the flows thus derived should have been forgotten in the intervening years, it is indeed strange that another method for the same purpose, which is very straightforward and simple, never seems to have been mentioned. In the following, this new method, which differs essentially from the geometrical method of Weingarten, will be presented together with some examples illustrating its practical applications.

For irrotational flow, the curl of the velocity vector is zero, and the velocity com-

*Received Dec. 2, 1954.

${ }^{1} \mathrm{~A}$. Wintner, On a geometrical method of deriving three-dimensional harmonic flows from two-dimensional ones, Quart. Appl. Math. 9, 102-105 (1951).

2,3See Ref. 1. 